\title{
Differentiating Among Children with PDD-NOS, ADHD, and those with a Combined Diagnosis on the Basis of WISC-III Profiles
}

\author{
J. G. M. Scheirs · E. A. Timmers
}

Published online: 22 October 2008

(c) The Author(s) 2008. This article is published with open access at Springerlink.com

\begin{abstract}
Pervasive Developmental Disorder-Not Otherwise Specified (PDD-NOS) and Attention Deficit/ Hyperactivity Disorder (ADHD) have partly overlapping symptoms. It can also be debated whether a third diagnostic category exists: children with a combined diagnosis. In this study an attempt was made to distinguish among the three groups on the basis of intelligence (WISC-III) profiles. It was found that the PDD-NOS group had higher verbal and performance IQ's, as well as higher WISC-III index scores than the ADHD group. Subtests Block Design and Mazes discriminated best. It was concluded that based on intelligence scores, only PDD-NOS and ADHD emerged as distinct categories, whereas the combined diagnosis did not. Future research on the distinctiveness of these diagnostic groups, however, should include variables other than IQ.
\end{abstract}

Keywords ADHD · PDD-NOS · WISC-III ·

Intelligence profiles

The prevalence of pervasive developmental disorders (PDDs) has been rising since the 1990s, with rates being reported now of about 60 per 10,000 children or even more (Chakrabarti and Fombonne 2001; Wing and Potter 2002; Baird et al. 2006). Of these children, at least half receive the diagnosis pervasive developmental disorder-not otherwise specified (PDD-NOS) (Chakrabarti and Fombonne 2001; Fombonne 2003). PDD-NOS refers to a

J. G. M. Scheirs $(\square)$ • E. A. Timmers

Developmental Psychology Section, Faculty of Social Sciences,

Tilburg University, P.O. Box 90153, 5000 LE Tilburg,

The Netherlands

e-mail: j.g.m.scheirs@uvt.nl pervasive developmental disorder that does not meet the criteria for any specific PDD, i.e. for autistic disorder, Rett's disorder, childhood's disintegrative disorder, or Asperger's disorder. Positive identifying characteristic of PDD-NOS are late onset, atypical symptomatology, and/or subthreshold symptomatology (American Psychiatric Association [APA] 2000).

The global definition of the concept PDD-NOS, which has also been referred to as a form of "mild autism", has given rise to diverse interpretations and may lead to low diagnostic reliability (Luteijn et al. 2000). Due to this vagueness, it is not surprising that there is considerable comorbidity of PDD-NOS with the symptoms of other childhood disorders, of which those of attention deficit/ hyperactivity disorder (ADHD) belong to the most striking ones (De Bruin et al. 2007). It has been shown that social and communication problems typical for PDD-NOS occur in children with ADHD, while the symptoms of inattention and hyperactivity/impulsivity characterizing ADHD also occur in children with PDD-NOS (e.g. Luteijn et al. 2000). Examples of clinical cases that had to be rediagnosed from ADHD to PPD-NOS (Jensen et al. 1997; Perry 1998), as well as children that evolved in the opposite direction as they matured (Fein et al. 2005), have confirmed that there are no sharp boundaries between the categories and that it is often difficult to diagnose children as belonging to either one.

The Diagnostic and Statistical Manual for Mental Disorders, Fourth Edition, Text Revision (DSM-IV-TR) (APA 2000) states that PDD broadly defined is one of the exclusion criteria for ADHD, and therefore that PDD-NOS and ADHD, despite of their overlapping symptoms and contrary to what can be seen in clinical practice, should not be diagnosed together. An increasing number of researchers, however, challenge the idea that PDD precludes 
ADHD and vice versa, and argue that a double diagnosis is justified in some cases (Frazier et al. 2001; Goldstein and Schwebach 2004; Ogino et al. 2005; Yoshida and Uchiyama 2004). It remains a prominent question, therefore, whether PDD-NOS, ADHD, and the combination of the two are distinct diagnostic categories.

Investigating intelligence profiles, i.e. comparing FullScale or Total (FIQ), Verbal (VIQ) and Performance (PIQ) IQ scores, or even comparing subtest score profiles among different diagnostic groups, is often done. It is common practice for two main reasons: better diagnosis of individual patients, and gaining more insight in the strengths and weaknesses that characterize a certain condition. It has been shown, however, that subtest profiles are less stable (i.e. less reliable) than profiles composed of IQ scores, or than profiles composed of the Wechsler index scores: Verbal Comprehension, Perceptual Organization, Processing Speed and Freedom from Distractibility (Livingston et al. 2003). This makes the subtest profile badly suited as an instrument in the diagnosis of a single patient. It is less problematic in research situations involving group comparisons, of course, since by calculating mean scores part of the random fluctuations present in the individual profiles are averaged out.

What is known until now about the IQ scores of the three diagnostic groups? Regarding ADHD, older versions of the Wechsler scales have generally pointed to lower intelligence scores in ADHD than in normal subjects (Schwean and Saklofske 1998). These results could not be corroborated on the basis of the WISC-III, however. On the basis of that test, normal average IQ scores tended to be seen and without consistent differences between performance and verbal IQ's (or between the perceptual organisation and verbal comprehension factor of the test) (Schwean and Saklofske 1998; Dickerson Mayes and Calhoun 2006).

Regarding individuals with autistic disorder, Lincoln et al. (1998), in a meta-analytic review, found the typical impairment of the verbal IQ's of autistic individuals relative to their performance IQ's. This VIQ $<$ PIQ pattern, however, could not be replicated by Siegel et al. (1996), nor by Dickerson Mayes and Calhoun (2003). It is known that about two-thirds of the autistic individuals are mentally retarded (Fombonne 2003; Baird et al. 2006). In subjects with PDD-NOS, cognitive impairments are in all likelihood less frequent and less severe (Chakrabarti and Fombonne 2001), although a lack of clarity still exists about the precise figures (Fombonne 2003; Baird et al. 2006). In many research reports including this one, only so called high-functioning patients (FIQ > 70) have been sampled. That is the reason that the reported Full-Scale IQ's are often not indicative of the level of intellectual functioning of the diagnostic groups as a whole.
Concerning PDD-NOS, only two publications exist that report on the verbal and performance IQ scores or subtest profiles of this group of individuals. Koyama et al. (2006) compared a group of Japanese children having PDD-NOS with a group of ADHD children. The authors found a VIQ $<$ PIQ pattern in the PDD-NOS group, which corroborated part of the literature on IQ differences in autistic individuals in general (Lincoln et al. 1998), while they found the reverse pattern in the children with ADHD, which was a new finding. De Bruin et al. (2006) also investigated a group of PDD-NOS children, but they didn't find a VIQ-PIQ discrepancy. These authors also investigated groups of children with autism and Asperger syndrome, respectively, and concluded that is was impossible to distinguish among the three groups on the basis of IQ scores.

The aim of this study was again to investigate the intelligence profiles (VIQ-PIQ) of children with PDD-NOS and ADHD, and to see whether the results of Koyama et al. (2006) could be replicated. A diagnostic group consisting of children with the combined diagnosis PDD-NOS plus ADHD was added. A second aim was to find out whether a distinction could be made among these three groups, based on the WISC-III-subtest scores of the participants.

\section{Method}

\section{Participants}

This study was based on archival data, obtained in the child and adolescent department of a large outpatient institution for mental health in the south of the Netherlands. The children were first seen in the institution during the period 2003-2007 and had been referred, mostly by general practitioners or youth care organizations, because of behavioural problems or psycho-social maladjustment displayed in school or at home. Children with mental retardation (FIQ $<70$ ) were generally not referred to this institution. Intelligence was not used in any way as a criterion for including cases in our study, however.

The total sample consisted of 115 children, 91 of whom were boys, and 24 were girls. Fifty-five children had been diagnosed as having PPD-NOS (75\% boys), 40 as having ADHD ( $80 \%$ boys), and 20 as having PPD-NOS plus ADHD (90\% boys). There were no significant differences among the groups with regard to $\operatorname{sex}\left(\chi^{2}(2)=2.15\right.$, $p=.34)$. The children were between 6 and 16 years of age. The mean age of the children was 9.7 years $(S D=2.8)$ for the total sample, while the mean age was 10.1 years for the PDD-NOS group, 9.6 years for the ADHD group, and 11.2 years for the combined diagnosis group, respectively. The range of IQ's observed (FIQ) was 
66-136 for PDD-NOS, 76-123 for ADHD, and 76-116 for the combined diagnosis.

The children had been diagnosed by clinical psychologists or youth psychiatrists after consultation of other health care professionals working in the same multidisciplinary team. The diagnoses were based on the developmental histories of the children as revealed from clinical interviews with the parents, and on observation as well as extended neuropsychological testing of the children themselves. For PDD-NOS and ADHD, the diagnoses were made according to the criteria in the DSM-IV-TR. The ADHD group comprised all children with this diagnosis, without regard to possible subtypes (predominantly inattentive, predominantly hyperactive-impulsive, or the combined type). Contrary to the DSM-IV-TR guidelines, but in accord with suggestions in the literature (Frazier et al. 2001; Goldstein and Schwebach 2004; Ogino et al. 2005; Yoshida and Uchiyama 2004), some of the children had been diagnosed with a combination of PDD-NOS and ADHD. A criterion for being included in the research groups was that PDD-NOS, ADHD or a combination of the two had to be the sole diagnosis. Children with psychiatric comorbidity or written suggestions thereof in their files were omitted from our samples.

Measures and Statistical Procedures

The Dutch version of the third edition of the Wechsler Intelligence Scale for Children (WISC-III ${ }^{\mathrm{NL}}$ ) had been administered to all children. This test consists of 13 subtests, the results of which can be combined into FIQ, VIQ (subtests Information, Arithmetic, Similarities, Vocabulary, Comprehension and Digit Span), PIQ (subtests Picture Completion, Substitution, Picture Arrangement, Block Design, Object Assembly, Symbol Search and Mazes), and the factor or index scores Verbal Comprehension Index (VCI), Perceptual Organisation Index (POI) and Processing Speed Index (PSI). The fourth factor Freedom from Distractibility did not emerge from an exploratory factor analysis on the data in a Dutch norm group (Kort et al. 2005). The quality of the norms of the Dutch version of this test, as well as its reliability and validity (construct validity) were judged to be satisfactory (Evers et al. 2000/2005).

Two-way analyses of variance with one between-subjects and one within-subjects factor were performed to test for differences among the diagnostic groups, for differences among the types of IQ scores (verbal versus performance IQ's, or the index scores), and for their interaction. Tukey/Kramer tests were used as post hoc tests. Profile analysis (Harris 2001, pp. 173-182) was done to test for equality of levels, flatness and parallelism of the WISC-III-subtest profiles for the three diagnostic groups. This was followed by a discriminant analysis, performed to predict group membership on the basis of the subtest scores. The level of significance was set at .05 for all tests and the degrees of freedom for testing of the repeated measurements factors were Greenhouse-Geisser corrected.

\section{Results}

Mean FIQ-scores and the corresponding standard deviations for the three groups, PDD-NOS, ADHD and the combined diagnosis, were $102.3 \quad(S D=12.0), 95.0$ $(S D=12.0)$, and $96.5(S D=11.8)$, respectively. VIQ and PIQ values for the three groups are shown in Fig. 1.

Analysis of variance carried out on the data in Fig. 1 (FIQ was omitted because of multicollinearity problems), showed a significant main effect of the factor Group: $F(2,112)=4.86, p=.01$. The main effect for the factor IQ-Type (levels verbal and performance IQ) was not significant, and neither was the interaction effect: $F(1,112)=$ $.37, p=.55$, and $F(2,112)=1.25, p=.29$, respectively.

Post hoc tests, carried out to examine the significant main effect further, revealed one significant pairwise difference: the mean IQ-scores of the PDD-NOS group were higher than those of the ADHD group $(Q=2.98, p=.01)$.

The mean values for the index scores VCI, POI and PSI are shown in Fig. 2. The means were calculated on the basis of those 112 subjects for whom the relevant data were complete. The outcomes of the statistical testing procedures (two-way repeated measures analysis of variance

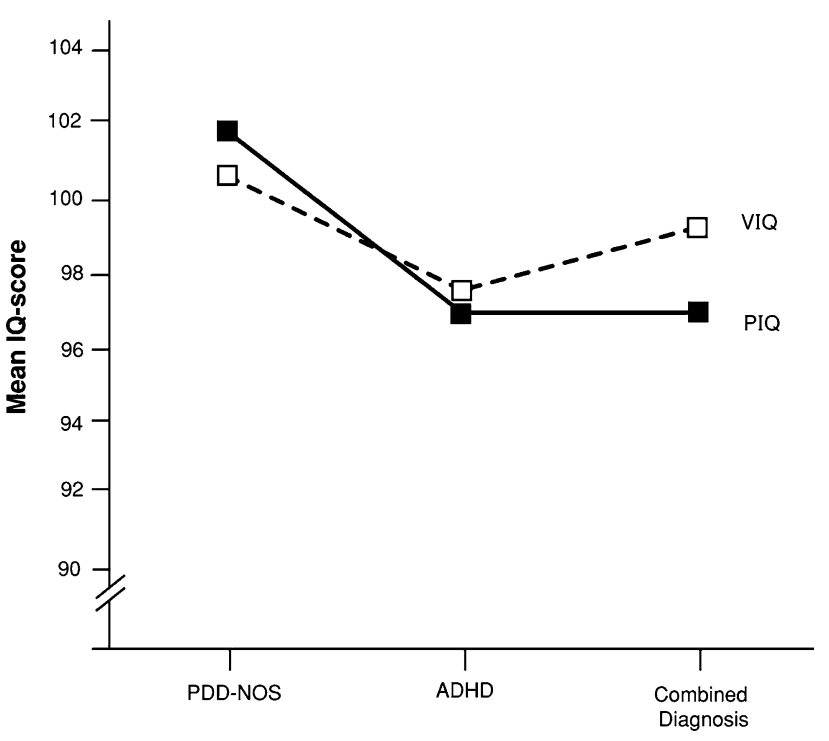

Fig. 1 Mean verbal (VIQ) and performance (PIQ) intelligence scores for the three diagnostic groups 


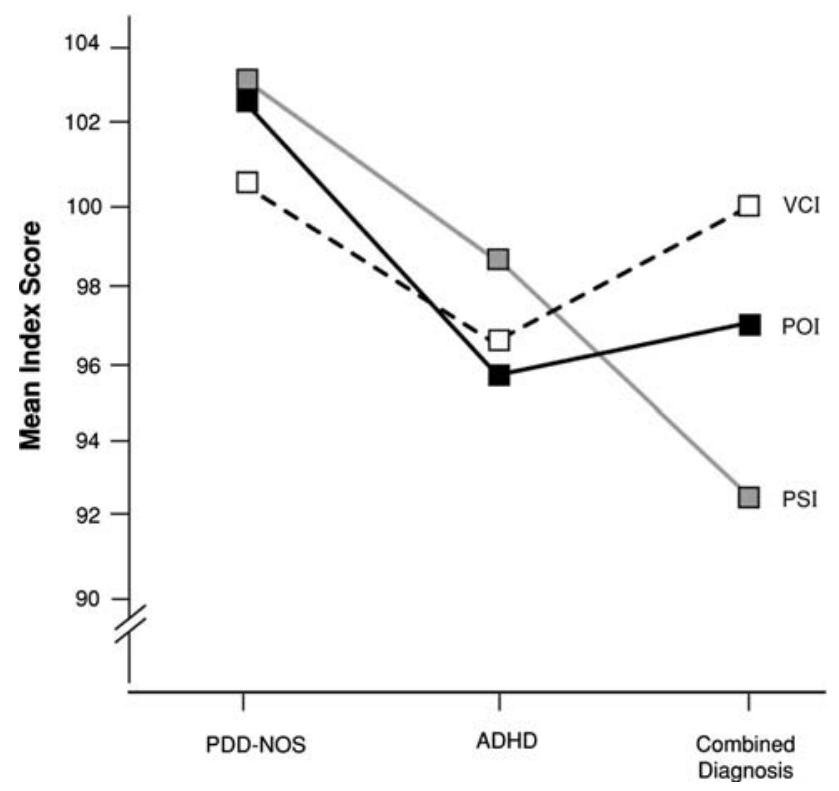

Fig. 2 Mean index scores for the three diagnostic groups. VCI: Verbal Comprehension Index. POI: Perceptual Organisation Index. PSI: Processing Speed Index

again, followed bij Tukey/Kramer post hoc tests) were comparable to the outcomes reported above. There was a significant main effect of Group: $F(2,109)=4.29$, $p=.02$. The main effect for the factor IQ-Type (levels VCI, POI and PSI) was not significant: $F(2,218)=.16$, $p=.84$. Post hoc tests for the Group main effect revealed a significant difference only between the index scores of the PDD-NOS group and those of the ADHD group $(Q=2.57$, $p=.03)$, the PDD-NOS group having the higher scores. Although the mean values in Fig. 2 indicate that the combined diagnosis group showed the largest differences among the index scores, such a conclusion was not supported by a test of the interaction effect, which was not statistically significant $(F(4,218)=1.69, p=.16)$.

The subtest profiles are presented in Fig. 3. These profiles were based on the data of those 106 children for whom the relevant data set was complete (PDD-NOS: $n=50$; ADHD: $n=37$; Combined Diagnosis: $n=19$ ). In a two-way mixed analysis of variance, the test for equality of levels (main effect for Group: $F(2,103)=$ $5.89, p=.00$ ), the test for flatness of the profiles (main effect for Subtest: $F(8.7,895)=1.83, p=.06)$, and the test for parallelism (Group $\times$ Subtest interaction: $F(17.4$, $895)=1.71, p=.04)$, were all significant or came close to it. These results corroborate what can be seen from Fig. 3 and what was already known from the IQ and index scores: that, overall, the PDD-NOS group attained the highest scores, while there was not much of a difference between the ADHD and the combined diagnosis groups. The differences between the PDD-NOS group on the one hand and the ADHD and the combined diagnosis group on the other, were most apparent in the performance part (right part) of the figure. Significant differences between the PDD-NOS group and one or both of the other two groups (i.e. non-overlapping 95\% confidence intervals) were present for the subtests Arithmetic, Picture Completion, Substitution, and Block Design.

To examine the group differences more closely, a direct discriminant analysis (all predictors entered simultaneously) was performed. The subtests were the predictors and group membership was the dependent variable.

Two discriminant functions were calculated. The $\chi^{2}(26)$-value for the two functions combined was 45.2 $(p=.01)$, but the second function was not significant. Therefore only the first function was interpreted. This function accounted for $76 \%$ of the between-group variability, and it separated the PDD-NOS group on the one hand from the ADHD and combined diagnosis group on the other. This can be seen in Fig. 4, where the function values for all the 106 children are graphed in a discriminant plane: the group means (centroids) differ primarily along the horizontal axis (function 1), while only small differences are present along the vertical axis (function 2). The subtests Block Design, Arithmetic and Mazes defined the first discriminant function. These subtests had the highest correlations with the function values: $r=.64, .52$ and .40 , respectively. Based on the standardized coefficients of this function, however, it could be concluded that in separating the PDD-NOS group from the other two groups, Block Design and Mazes were the most important variables and that Arithmetic didn't contribute much, given the other two predictors in the set. The outcome of this statistical procedure is also reflected in Fig. 3, showing that the highest scores for the subtests Arithmetic, Block Design and Mazes were attained in the PDD-NOS group.

The classification procedure of the discriminant analysis revealed that, with equal prior probabilities for the groups, $60 \%$ of the cases $(66 \%$ of PPD-NOS, 54\% of ADHD, and $58 \%$ of the combined diagnosis group) could be classified correctly, against a $33 \%$ correct classification rate due to chance. This was a significant improvement $(z=5.92$, $p=.00)$.

When in a separate discriminant analysis PDD-NOS was compared to ADHD only, it appeared that Block Design remained the subtest with the highest discriminating value (significance of function: $p=.00$; correlation of subtest with function: $r=.56$ ). In an analysis comparing PDDNOS to the combined diagnosis only, Substitution and Block Design defined the discriminant function (significance of function: $p=.04$; correlations of subtest with function: $r=.66$ and $r=.59$, respectively). 
Fig. 3 Mean WISC-III subtest scores for the three diagnostic groups. The asterisks indicate subtests for which the $95 \%$ confidence intervals (of the PDD-NOS group and either one or both of the other groups) did not overlap

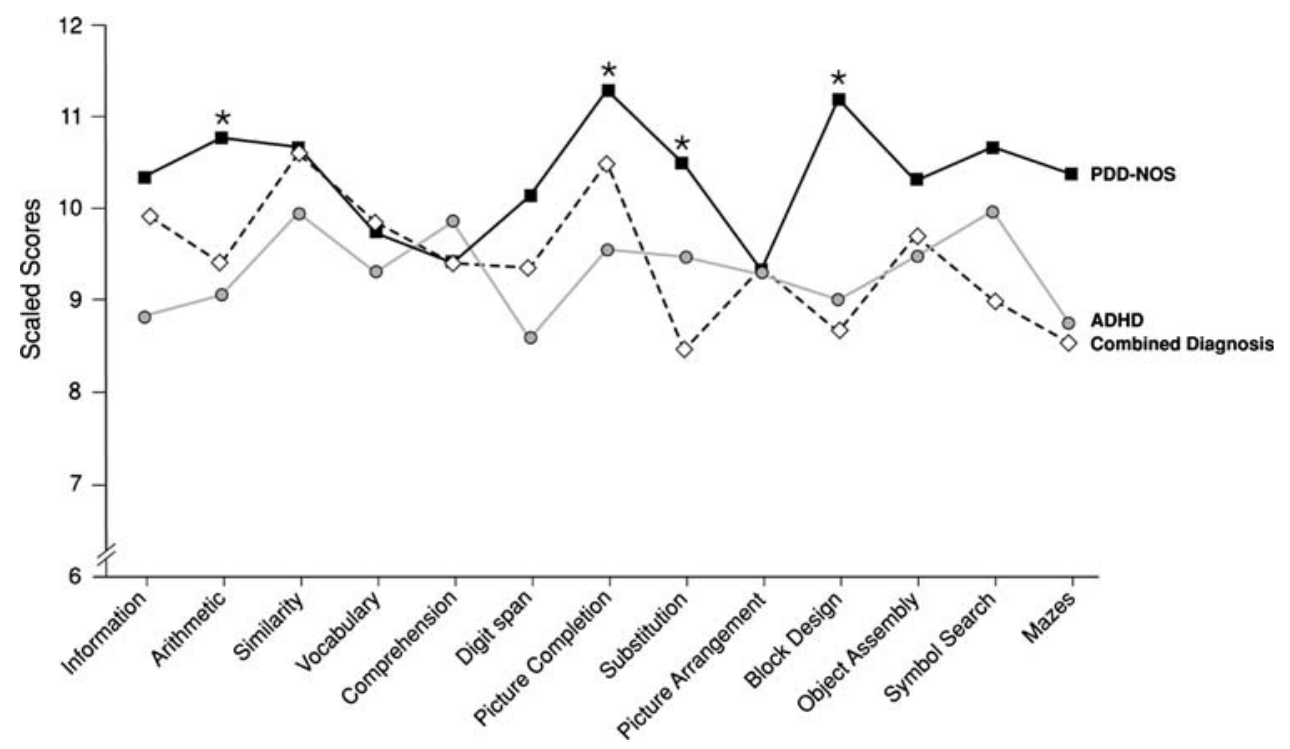

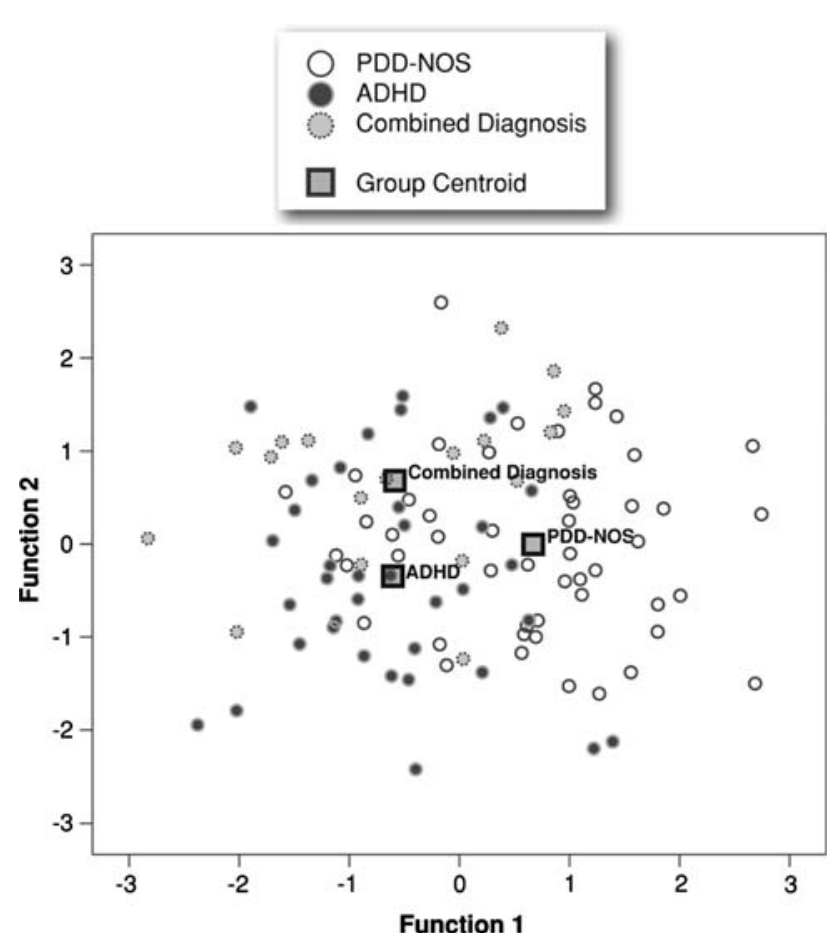

Fig. 4 Individual values of $n=106$ children and group means (centroids) on two discriminant functions, showing the separability of the three groups on the basis of WISC-III subtest scores

\section{Discussion}

PDD-NOS is a frequently occurring disorder, and also one of the least investigated categories of the pervasive developmental disorders. There is a partial overlap of its symptoms with those of another important childhood disorder, ADHD. The questions that have arisen are 1., whether PDD (broadly defined) and ADHD are really discrete syndromes (Hattori et al. 2006), and 2., if this is so, whether a combined diagnosis of PDD-NOS and ADHD is ever justified (Luteijn et al. 2000). To shed more light on these questions, we investigated whether children diagnosed as having PDD-NOS or ADHD, and children meeting the criteria for both diagnoses could be distinguished on the basis of their intelligence profiles. A further research aim was to determine how our results would compare to the earlier research outcomes of De Bruin et al. (2006) and Koyama et al. (2006).

We found that mean WISC IQ scores (Full scale, Verbal, and Performance IQ) and the index scores (VCI, POI, and PSI) did differ among the three groups. The pattern was the same for all measures: the PDD-NOS group attained the highest scores, whereas the scores of the ADHD and combined diagnosis groups were lower and highly similar to one another. Statistically significant differences were found only between the PDD-NOS and the ADHD group. These results suggest that the first question above can be answered positively: the distinction between the PDD-NOS and ADHD diagnoses can be validated on the basis of IQ scores. On the basis of IQ scores alone, however, the existence of a distinct combined diagnosis group could not be warranted.

These are merely suggestive conclusions, of course. Firstly, a statistical test not coming up with a significant difference does not imply that a difference does not exist. A lack of power to detect such a difference might be due to small sample sizes, and we didn't have many subjects in the combined diagnosis group. Secondly, as Schwean and Saklofske (1998) have argued, ADHD research in general suffers from methodological weaknesses, of which inconsistent subject selection criteria is undoubtedly the most serious one. For PDD-NOS, being a left-over diagnostic category, and for a combined diagnosis group, the situation 
might be even worse. The outcome of a particular study might therefore depend on the way the subjects were selected and on how the diagnoses were made. To make replications possible and to increase the generalizability of research findings, we should rather use standardized and quantitative measures than clinical judgements to base our diagnoses on (e.g. Volkmar et al. 2004). The fact that objective measures of symptom behaviors were missing in this study is a serious limitation.

Up to this date, only two articles described the profiles of cognitive functioning in children with PDD-NOS (De Bruin et al. 2006; Koyama et al. 2006). The latter of these two also made a comparison with ADHD children. The inclusion in the present study of a group of children with a combined diagnosis seems to be new.

De Bruin and coworkers did not find a VIQ-PIQ discrepancy in the PDD-NOS children of their study. Koyama and coworkers did, PIQ scores being larger than VIQ scores in the PDD-NOS group, while the reverse pattern was found in children diagnosed with ADHD. Our mean results, although small and far from significant, showed discrepancies in the same direction. Siegel et al. (1996) have argued that the presence of a VIQ-PIQ difference in autism is severity dependent, i.e. that such a pattern is most likely to show up in individuals with low cognitive abilities. PDD-NOS individuals had a mean FIQ-score of 90 in the De Bruin study, of 95 in the Koyama study, and of 102 in the present study. The PDD-NOS individuals investigated in these studies thus functioned at an average or just below average level of ability. In the present research at least, the PDD-NOS group was selective in that it did not contain low functioning individuals. It remains to be seen, therefore, whether a VIQ-PIQ discrepancy is a true characteristic of the total population of PDD-NOS children.

When distinguishing the groups on the basis of subtest profiles, the discriminant analysis revealed that Block Design and Mazes were the most important variables. PDD-NOS children in our study scored higher on these two subtests than did the other two groups. The PDD-NOS children also attained very high scores on Block Design in an absolute sense. The Block Design advantage (but not the higher scores on Mazes) for PDD-NOS as compared to ADHD children was also found by Koyama et al. (2006). For high-functioning autistic children, the good performance on Block Design (as compared to other subtests) has even been found to be an almost universal characteristic; low scores for both Picture Arrangement and Comprehension were frequently found in these subjects at the same time (Siegel et al. 1996). The fact that this particular pattern of subtest scores was present in our PDD-NOS group strengthens the credibility of the findings and the accuracy of the PDD-NOS diagnoses. Absolute good performance by PDD-NOS children on Mazes (but not on Block Design this time) was found by De Bruin et al. (2006). It can be concluded that in the IQ range of above 70, PDD-NOS children perform better than ADHD children and those with a combined diagnosis, which can primarily be attributed to differences in visuospatial and visuomotor abilities among the groups.

In this study we added to the knowledge about children with PDD-NOS, which is a disorder with a high prevalence rate but with little information about intelligence and intelligence profiles published to date. We compared the intelligence scores of the PDD-NOS group with those of an ADHD group and a group with a combined diagnosis. It appeared that on the basis of intelligence scores alone, a distinction could be made between PDD-NOS and ADHD. The combined diagnosis group did not emerge as a group with distinct characteristics and resembled the ADHD group most. This does not prove, of course, that the combined diagnosis in itself is not a valid category. It only says that intelligence couldn't tell it apart from the other two. To better understand the distinctiveness of the three diagnoses, future research should address other variables too. Preferably these should be variables more closely related to the core symptoms of PDD-NOS and ADHD, i.e. hyperactivity and problems in attention, social functioning and communication.

There are two further recommendations that could increase the sensitivity of future research. First, if possible, one should distinguish between the three subtypes of ADHD, as described in the DSM-IV-TR. Diamond (2005) argued that the inattentive subtype without hyperactivity is a disorder distinct from ADHD including hyperactivity. She argued that differences exist in the abilities and test performance of these children, among which are differences in language and arithmetic skills. It is reasonable to assume then, that ADHD subtypes differ also with regard to WISC-III profiles. The distinction might also relate to the fact that in the present study no differences were found between the ADHD and the combined diagnosis group. Diamond concluded that children of the inattentive type but without hyperactivity tend to be socially isolated and withdrawn. These are also the main characteristics of PDD-NOS (Buitelaar and Van der Gaag 1998). If confusion or overlap regarding PDD-NOS and ADHD diagnoses exists, this might therefore mainly concern the inattentive subtype of ADHD. As a consequence of this, we would like to speculate that children who receive an ADHD diagnosis (unspecified) or a combined diagnosis are predominantly those that show hyperactivity as one of their symptoms and that therefore these two groups are more alike. Of course, additional research using quantified measures of hyperactivity is necessary to prove or disprove this.

As a second recommendation for follow-up research, it should be ascertained whether ADHD children receive 
pharmacological treatment at the moment of testing. Stimulant medication has been found to increase cognitive performance of ADHD children, especially their cognitive flexibility, attention and vigilance (Pietrzak et al. 2006). Although direct effects of stimulant medication on WISCIII scores were not found in a few older studies described by Schwean and Saklofske (1998), these authors withheld final judgement and indicated that medication effects are probably dose dependent. ADHD children thus actually may benefit from medication when IQ tested. In the present study, the ADHD children were tested at their entrance into the institution and at the beginning of the diagnostic process. At that moment, they were in all likelihood still untreated. This provided us with a clearer picture of differences in IQ performance than when IQ testing had taken place after the diagnosis had been made.

Acknowledgments The authors are indebted to the Institution for Mental Health in Eindhoven (GGzE) for making this research possible. For their inspiring ideas, clinical psychologists Marjan Veldhoen-Steutel and Miriam Nijkamp-Geertman deserve special mention. Yvonne Janssens prepared the illustrations.

Open Access This article is distributed under the terms of the Creative Commons Attribution Noncommercial License which permits any noncommercial use, distribution, and reproduction in any medium, provided the original author(s) and source are credited.

\section{References}

American Psychiatric Association. (2000). Diagnostic and statistical manual of mental disorders, text revision (4th ed.). Washington, DC: Author.

Baird, G., Simonoff, E., Pickles, A., Chandler, S., Loucas, T., Meldrum, D., et al. (2006). Prevalence of disorders of the autism spectrum in a population cohort of children in South Thames: The Special Needs and Autism Project (SNAP). Lancet, 368, 210-215. doi:10.1016/S0140-6736(06)69041-7.

Buitelaar, J. K., \& Van der Gaag, R. J. (1998). Diagnostic rules for children with PDD-NOS and multiple complex developmental disorder. Journal of Child Psychology and Psychiatry and Allied Disciplines, 39, 911-919. doi:10.1017/S0021963098002820.

Chakrabarti, S., \& Fombonne, E. (2001). Pervasive developmental disorders in preschool children. Journal of the American Medical Association, 285, 3093-3099. doi:10.1001/jama.285.24.3093.

De Bruin, E. I., Ferdinand, R. F., Meester, S., De Nijs, P. F. A., \& Verheij, F. (2007). High rates of psychiatric co-morbidity in PDD-NOS. Journal of Autism and Developmental Disorders, 37, 877-886. doi:10.1007/s10803-006-0215-x.

De Bruin, E. I., Verheij, F., \& Ferdinand, R. F. (2006). WISC-R subtest but no overall VIQ-PIQ difference in Dutch children with PDD-NOS. Journal of Abnormal Child Psychology, 34, 263271. doi:10.1007/s10802-005-9018-3.

Diamond, A. (2005). Attention-deficit disorder (attention-deficit/ hyperactivity disorder without hyperactivity): A neurobiologically and behaviorally distinct disorder from attention-deficit/ hyperactivity disorder (with hyperactivity). Development and Psychopathology, 17, 807-825. doi:10.1017/S0954579405050388
Dickerson Mayes, S., \& Calhoun, S. L. (2003). Analysis of WISC-III, Stanford-Binet:IV, and academic achievement test scores in children with autism. Journal of Autism and Developmental Disorders, 33, 329-341. doi:10.1023/A:1024462719081.

Dickerson Mayes, S., \& Calhoun, S. L. (2006). WISC-IV and WISCIII profiles in children with ADHD. Journal of Attention Disorders, 9, 486-493. doi:10.1177/1087054705283616.

Evers, A., Van Vliet-Mulder, J. C., \& Groot, C. J. (2000). Documentatie van tests en testresearch in Nederland (Suppl. 2005/03). Assen: Van Gorcum \& Comp.

Fein, D., Dixon, P., Paul, J., \& Levin, H. (2005). Brief report: Pervasive developmental disorder can evolve into ADHD: Case illustrations. Journal of Autism and Developmental Disorders, 35, 525-534. doi:10.1007/s10803-005-5066-3.

Fombonne, E. (2003). Epidemiological surveys of autism and other pervasive developmental disorders: An update. Journal of Autism and Developmental Disorders, 33, 365-382. doi: 10.1023/A:1025054610557.

Frazier, J. A., Biederman, J., Bellordre, C. A., Garfield, S. B., Geller, D. A., Coffey, B. J., et al. (2001). Should the diagnosis of attention-deficit/hyperactivity disorder be considered in children with pervasive developmental disorder? Journal of Attention Disorders, 4, 203-211. doi:10.1177/108705470100400402.

Goldstein, S., \& Schwebach, A. J. (2004). The comorbidity of pervasive developmental disorder and attention deficit hyperactivity disorder: Results of a retrospective chart review. Journal of Autism and Developmental Disorders, 34, 329-339. doi:10.1023/ B:JADD.0000029554.46570.68.

Harris, R. J. (2001). A primer of multivariate statistics (3rd ed.). Mahwah, NJ: Erlbaum.

Hattori, J., Ogino, T., Abiru, K., Nakano, K., Oka, M., \& Ohtsuk, Y. (2006). Are pervasive developmental disorders and attentiondeficit/hyperactivity disorder distinct disorders? Brain and Development, 28, 371-374. doi:10.1016/j.braindev.2005.11.009.

Jensen, V. K., Larrieu, J. A., \& Mack, K. K. (1997). Differential diagnosis between attention-deficit/hyperactivity disorder and pervasive developmental disorder-not otherwise specified. Clinical Pediatrics, 36, 555-561. doi:10.1177/000992289703601001.

Kort, W., Schittekatte, M., Dekker, P. H., Verhaeghe, P., Compaan, E. L., Bosmans, M., et al. (2005). WISC-III ${ }^{N L}$. Handleiding en verantwoording. Nederlandse bewerking. London: Harcourt Test Publishers.

Koyama, T., Tachimori, H., Osada, H., \& Kurita, H. (2006). Cognitive and symptom profiles in high-functioning pervasive developmental disorder not otherwise specified and attention-deficit/ hyperactivity disorder. Journal of Autism and Developmental Disorders, 36, 373-380. doi:10.1007/s10803-006-0075-4.

Lincoln, A., Courchesne, E., Allen, M., Hanson, E., \& Ene, M. (1998). Neurobiology of Asperger syndrome. Seven case studies and quantitative magnetic resonance imaging findings. In E. Schopler \& G. B. Mesibov (Eds.), Asperger syndrome or highfunctioning autism? (pp. 145-163). New York: Plenum Press.

Livingston, R. B., Jennings, E., Reynolds, C. R., \& Gray, R. M. (2003). Multivariate analyses of the profile stability of intelligence tests: High for IQs, low to very low for subtest analyses. Archives of Clinical Neuropsychology, 18, 487-507. doi:10.1016/S0887-6177(02)00147-6.

Luteijn, E. F., Serra, M., Jackson, S., Steenhuis, M. P., Althaus, M., Volkmar, F., et al. (2000). How unspecified are disorders of children with a pervasive developmental disorder not otherwise specified? A study of social problems in children with PDD-NOS and ADHD. European Child and Adolescent Psychiatry, 9, 168-179. doi:10.1007/s007870070040.

Ogino, T., Hattori, J., Abiru, K., Nakano, K., Oka, E., \& Ohtsuka, Y. (2005). Symptoms related to ADHD observed in patients with 
pervasive developmental disorder. Brain and Development, 27, 345-348. doi:10.1016/j.braindev.2004.09.006.

Perry, R. (1998). Misdiagnosed ADD/ADHD; rediagnosed PDD. Journal of the American Academy of Child and Adolescent Psychiatry, 37, 113-114.

Pietrzak, R. H., Mollica, C. M., Maruff, P., \& Snyder, P. J. (2006). Cognitive effects of immediate-release methylphenidate in children with attention-deficit/hyperactivity disorder. Neuroscience and Biobehavioral Reviews, 30, 1225-1245. doi:10.1016/ j.neubiorev.2006.10.002.

Schwean, V. L., \& Saklofske, D. H. (1998). WISC-III assessment of children with attention deficit/hyperactivity disorder. In A. Prifitera \& D. H. Saklofske (Eds.), WISC-III. Clinical use and interpretation. Scientist-practitioner perspectives (pp. 91-118). San Diego: Academic Press.

Siegel, D. J., Minshew, N. J., \& Goldstein, G. (1996). Wechsler IQ profiles in diagnosis of high-functioning autism. Journal of
Autism and Developmental Disorders, 26, 389-406. doi:10.1007/ BF02172825.

Volkmar, F. R., Lord, C., Bailey, A., Schultz, R. T., \& Klin, A. (2004). Autism and pervasive developmental disorders. Journal of Child Psychology and Psychiatry and Allied Disciplines, 45, 135-170. doi:10.1046/j.0021-9630.2003.00317.x.

Wing, L., \& Potter, D. (2002). The epidemiology of autistic spectrum disorders: Is the prevalence rising? Mental Retardation and Developmental Disabilities Research Reviews, 8, 151-161. doi: 10.1002/mrdd.10029.

Yoshida, Y., \& Uchiyama, T. (2004). The clinical necessity for assessing attention deficit/hyperactivity disorder (AD/HD) symptoms in children with high-functioning pervasive developmental disorder (PDD). European Child and Adolescent Psychiatry, 13, 307-314. doi:10.1007/s00787-004-0391-1. 